\title{
Persistencia de los efectos de la descompactación del suelo con paratill
}

\author{
Perdomo, Luis Ignacio'; Martin Rollhauser ${ }^{1}$; Esteban Melani ${ }^{2}$; Facundo Guilino ${ }^{1}$; Laura \\ Mónica Draghi ${ }^{1}$; Daniel Jorajuría ${ }^{1}$; Guillermo Oliverio Sarli' ${ }^{1}$; Rafael Villarreal' ${ }^{1}$; Luis \\ Lozano $^{1}$; Carlos Germán Soracco ${ }^{1}$; Mirta García'; Telmo Palancar ${ }^{1,3}$
}

${ }^{1}$ Centro de Investigación de Suelos para la Sustentabilidad Agropecuaria y Forestal (CISSAF), Facultad de Ciencias Agrarias y Forestales, Universidad Nacional de La Plata, Buenos Aires, Argentina; ${ }^{2}$ Agencia De Extensión Rural Chascomus (AER Chascomus), Instituto Nacional de Tecnología Agropecuaria (INTA), Buenos Aires, Argentina; ${ }^{3}$ telmo@agro.unlp.edu.ar

Perdomo, Luis Ignacio; Martin Rollhauser; Esteban Melani; Facundo Guilino; Laura Mónica Draghi; Daniel Jorajuría; Guillermo Oliverio Sarli; Rafael Villarreal; Luis Lozano; Carlos Germán Soracco; Mirta García; Telmo Palancar (2020) Persistencia de los efectos de la descompactación del suelo con paratill. Rev. Fac. Agron. Vol 119 (1): 1-7. https://doi.org/10.24215/16699513e035

\begin{abstract}
En la Argentina predomina como sistema productivo la siembra directa donde la ausencia de remoción y el tránsito de máquinas cada vez más pesadas han incrementado los problemas de compactación. Para revertir este fenómeno se utilizan descompactadores. Existen datos dispares de cuán prolongada es la duración del efecto de descompactación. Se realizó un ensayo utilizando un descompactador angulado lateral (Paratill) en un suelo Hapludol Thapto Árgico. A los dos años se evaluó la resistencia a la penetración, la densidad aparente, la infiltración y la producción de materia seca comparando el tratamiento descompactado con el testigo (sin descompactar). No se encontraron diferencias en densidad aparente ni en infiltración entre ambos tratamientos. Hubo diferencias en la resistencia a la penetración donde el tratamiento testigo alcanzó valores mayores al descompactado en parte del perfil trabajado y aún debajo de la profundidad máxima alcanzada. La materia seca producida en el tratamiento descompactado duplicó a la del testigo evidenciando que el efecto de la descompactación se mantiene dos años después de realizada. Se concluye que el efecto de la descompactación se evidencia aún dos años después de efectuada en el parámetro resistencia a la penetración y que el cultivo percibe estos efectos
\end{abstract}

Palabras Clave: compactación del suelo, resistencia a la penetración, densidad aparente, infiltración, producción de materia seca

Perdomo, Luis Ignacio; Martin Rollhauser; Esteban Melani; Facundo Guilino; Laura Mónica Draghi; Daniel Jorajuría; Guillermo Oliverio Sarli; Rafael Villarreal; Luis Lozano; Carlos Germán Soracco; Mirta García; Telmo Palancar (2020) Soil loosening persistence with paratill. Rev. Fac. Agron. Vol 119 (1): 1-7. https://doi.org/10.24215/16699513e035

In Argentina, no-tillage is the main management system where the absence of soil disturbance and the traffic of heavy machine has increased the problems of soil compaction. In order to reverse this phenomenon, decompaction is used, with contradictory results on the duration of the effect of soil loosing. The study was performed using a lateral angled decompactor (Paratill) in a Thapto Argic Hapludol. After two years, penetration resistance, bulk density, infiltration and dry matter production was evaluated under two treatments: decompacted; and control (without decompaction). No differences were found in bulk density or infiltration between treatments. Along the studied soil profile, the control treatment showed higher values of penetration resistance in comparison with the decompacted only between 7 and $30 \mathrm{~cm}$, even below the maximum depth reached. Dry matter was higher under decompaction treatment, showing that the effect of decompaction was maintained two years after its application. In conclusion, the effect of decompaction is still evident after two years, improving the penetration resistance parameter and crop biomass production.

Keywords: soil compaction, penetration resistance, bulk density, infiltration, dry matter production

https://revistas.unlp.edu.ar/revagro

Recibido: 05/01/2019

Aceptado: 10/06/2019

Disponible on line: $01 / 07 / 2020$

ISSN 0041-8676 - ISSN (on line) 1669-9513, Facultad de Ciencias Agrarias y Forestales, UNLP, Argentina 


\section{INTRODUCCIÓN}

En la República Argentina la mayor parte de la superficie cultivada se realiza bajo el sistema de Siembra Directa (SD) ocupando el $91 \%$ de la superficie total agrícola (Nocelli, 2017). La adopción masiva del sistema de siembra directa disminuyó la intensidad del tráfico agrícola en cuanto al número de pasadas pero no en cuanto a la relación masa/eje que el suelo deformable debe soportar. El suelo se compacta cada vez que las tensiones aplicadas por el tránsito causan daños y cambios en la proporción del espacio poroso, la distribución de tamaños de poros, la relación de vacíos, la continuidad de los poros y la impedancia del suelo (O'Sullivan et al., 1999). Este problema es cada vez más pronunciado debido al aumento del ancho de labor de los implementos para obtener una mayor capacidad de trabajo, y consecuentemente el aumento de peso tanto de implementos como de tractores para lograr la eficiencia tractiva global óptima (Botta et al., 2009a). Es una problemática compleja, y el uso de parámetros tan simples como la densidad aparente, a menudo resultan deficientes para evaluar los daños producidos (Botta et al., 2016).

En la región pampeana, la ausencia de remoción, la presencia de maquinaria agrícola de mayor peso y el tránsito en húmedo, generaron un aumento en la compactación superficial y subsuperficial del suelo. Por ello, existe un interés creciente en revertir esta limitante a la productividad de los cultivos (Elisei et al., 2012). Según da Silveira Nicoloso et al. (2008) la compactación es una de las principales causas de la disminución del rendimiento en los suelos agrícolas. Balbuena et al. (1998) recomiendan el uso de descompactadores como una práctica común para intentar revertir este fenómeno.

La labranza profunda reduce el tamaño de los bloques estructurales e incrementa la infiltración al aumentar el volumen de los macroporos. Spoor et al. (2003) recomiendan que el trabajo de descompactación del suelo sea de fisuramiento y no de roturación, priorizando así la persistencia de la labor en el tiempo al no disminuir su capacidad portante ante nuevas cargas ejercidas por el paso posterior de máquinas. En este sentido, Botta et al. (2010) concluyeron que los efectos del subsolado duran dos años y los del cincelado uno. Terminiello et al. (2007) evaluaron los efectos del tránsito de un conjunto tractor - sembradora sobre sectores del terreno descompactados y sin descompactar (testigo) bajo un sistema de siembra directa, encontrando que en el sector descompactado los efectos de la descompactación perduraron durante todo el ciclo del cultivo. Vepraskas et al. (1995), encontraron que el efecto residual del subsolado sobre el crecimiento de raíces, se mantenía alrededor de los dos años posteriores a la labranza, para desaparecer al tercer año como consecuencia de la recompactación. Busscher et al. (1986) afirman que el efecto del subsolado se manifiesta en las etapas tempranas del crecimiento de las raíces de cultivos, ya que posteriormente se produce la recompactación de las capas aflojadas. Barber (1994) en un ensayo de 4 años de duración, observó que la recompactación posterior a la labranza, no afectaba el crecimiento de raíces tan severamente como el suelo compactado previo a la labranza.

La problemática de compactación es detectada mediante mermas en el rendimiento, menor exploración de raíces, mayor densidad aparente, menor infiltración, por lo cual debe ser monitoreada a través de varios parámetros indicadores de la modificación de las condiciones mecánicas, físicas y químicas del suelo afectado (Hamza \& Anderson, 2005). Para caracterizar el efecto del tránsito sobre la matriz del suelo, la utilización de las variables experimentales resistencia a la penetración y densidad aparente están extensamente difundidas (Alakukku, 1997). Sin embargo, las propiedades indicadoras de retención de agua en el suelo, tamaño y distribución de los poros y resistencia a la penetración, son variables más sensibles para evidenciar la compactación que la densidad aparente (Alakukku, 1996; Botta et al., 2009b).

Elisei et al. (2012) trabajando con montantes angulados rectos y angulados curvos encontraron diferencias significativas en los valores de índice de cono (IC) con respecto al testigo, sin labor de escarificado. Álvarez et al. (2010) realizaron ensayos en suelos de la pampa ondulada en los que se efectuó una labor de descompactación a una profundidad de 0,35 $\mathrm{m}$ y encontraron valores de resistencia a la penetración menores que en los testigos sin descompactar. Sobre suelos del sur de Córdoba, clasificados como Haplustoles y Hapludoles, Cholaky et al. (2009) realizaron la labor de descompactación con escarificadores de rejas aladas a una profundidad de 0,27 m. Los mismos encontraron, en todos los casos, que la resistencia a la penetración fue estadísticamente menor que en el tratamiento testigo a la profundidad labrada. Colareda (2013) evaluó la resistencia a la penetración en un Argiudol típico luego del pasaje de un escarificador de montantes de lámina curva hasta una profundidad máxima de $0,35 \mathrm{~m}$, encontró significancia en este parámetro con respecto al testigo hasta una profundidad de 0,5 m. Hilbert \& Pincu (2000), trabajaron sobre un suelo Arguidol ácuico hasta una profundidad máxima de $0,30 \mathrm{~m}$, y encontraron significancia con respecto al testigo en la resistencia a la penetración hasta $0,42 \mathrm{~m}$ de profundidad.

Respecto a valores limitantes de resistencia a la penetración para el crecimiento de raíces, Threadgill (1982) indicó que valores de 1,5 MPa disminuyen el crecimiento de raíces y valores de 2,0 MPa lo impiden. En lo que respecta a la densidad aparente, Daddow \& Warrington (1983) establecieron que $1,65 \mathrm{Mg} / \mathrm{m}^{3}$ es el umbral de densidad aparente para permitir el crecimiento radicular. Los valores críticos de la densidad aparente para el crecimiento de las raíces varían según la textura que presenta el suelo y de la especie que se trate. De acuerdo a Jones (1983) para suelos arenosos una densidad aparente de 1,759 $\mathrm{Mg} / \mathrm{m}^{3}$ limita el crecimiento de las raíces de girasol, mientras que en suelos arcillosos ese valor crítico es de 1,460 a $1,630 \mathrm{Mg} / \mathrm{m}^{3}$ para la misma especie.

Respecto al parámetro infiltración, la clase textural, el uso y el manejo cultural del lote son los factores que mayor influencia tienen sobre su tasa, de acuerdo a del Barrio (1984). Alakukku (1996), reporta reducciones significativas de la conductividad hidráulica sobre la huella producto de la compactación del suelo efectuada 
por los vehículos, en comparación con las áreas no disturbadas, lo cual fue asociado con un incremento de la densidad aparente y un decrecimiento de la macroporosidad. Soracco et al. (2005) hallaron densidades de 0,$77 ; 1,15$ y $1,43 \mathrm{Mg} / \mathrm{m}^{3}$ en 3 sectores de un lote con diferentes grados de trabajo mecánico y pisoteo animal: recién labrado para huerta, con una pastura de 8 años de antigüedad y con un elevado pisoteo animal respectivamente. La infiltración en los 3 sectores fue inversamente proporcional a la densidad evaluada, teniendo el suelo recién labrado la mayor infiltración. Álvarez et al. (2006) evaluaron el efecto de la descompactación con un subsolador en suelo bajo siembra directa sobre algunas propiedades físicas del suelo, a la siembra y a la cosecha del cultivo de maíz. La densidad aparente no mostró variaciones significativas a la siembra y a la cosecha. Respecto a la variable infiltración, la descompactación antes de la siembra influyó notablemente sobre el ingreso del agua al suelo, pero la misma no se mantuvo en el tiempo y al momento de la cosecha los valores de infiltración no mostraron diferencias significativas con el testigo sin subsolado.

Existen antecedentes sobre reducción en el rendimiento por causas de

compactación (Tolon-Becerra et al., 2011; Marinello et al., 2017). Jorajuría et al. (1997) encontraron una disminución significativa de materia seca de una pastura atribuible a la compactación inducida.

El objetivo general del presente trabajo fue evaluar la perdurabilidad de una labor de descompactación a través de la medición de distintas variables físicas y biológicas.

\section{MATERIALES Y MÉTODOS}

El ensayo se realizó sobre un suelo de textura franca clasificado como Hapludol Thapto Árgico (Soil Taxonomy) (INTA, 2010), de la serie Los Naranjos y con una capacidad de uso Ilw. El mismo se encuentra ubicado en el establecimiento de producción agrícolaganadera "La Polvorilla" (coordenadas $36^{\circ}$ 01' 31,8" Latitud Sur y $57^{\circ} 48^{\prime} 16,6$ " Longitud Oeste), en el partido de Castelli, Provincia de Buenos Aires. Durante el año 2015, se realizó un tratamiento de descompactación con un implemento de montantes angulados laterales ("Paratill") hasta una profundidad de $25 \mathrm{~cm}$ sobre un lote ganadero con implantación de pasturas y verdeos. En el año 2009 se implantó una pastura de alfalfa (Medicago sativa) que duró 3 años y, en el año 2012, se implantó como verdeo de invierno raigrás (Lolium perenne) y maíz (Zea mays) para silaje. En el año 2013 y 2014 se repitió la secuencia de cultivos del año 2012.

El ensayo fue diseñado en 4 bloques aleatorizados contando con dos tratamientos: 1) Pasaje de Paratill (P) y 2) Testigo (sin Paratill, $T$ ). La labor de descompactación se realizó el día 14/3/2015, luego del picado de maíz para silaje. En marzo de 2015 se realizó la siembra de una pradera de alfalfa para pastoreo directo en ambos tratamientos. Las parcelas fueron sometidas a pastoreo directo.

Luego de transcurridos dos años de la descompactación, se evaluaron parámetros que permitieran visualizar la persistencia del efecto de dicha labor en algunas propiedades físicas del suelo y su posible respuesta en el cultivo.

Se determinó la resistencia a la penetración mediante un penetrómetro de cono electrónico SC900 Soil Compaction Meter Fieldscout, construido bajo Norma ASAE S.313, con principio de medición de profundidad por ultrasonido. Los valores de índice de cono fueron agrupados en rangos de profundidad de $2,5 \mathrm{~cm}$ y se registraron hasta los $35 \mathrm{~cm}$ de profundidad. Se efectuaron 45 repeticiones por cada rango de profundidad y por cada tratamiento.

Los datos de índice de cono fueron analizados en referencia a la humedad gravimétrica del suelo. La humedad gravimétrica se obtuvo por diferencia de peso de muestras de suelo húmedo y seco. El secado se realizó en estufa a $105^{\circ} \mathrm{C}$ durante $24 \mathrm{hs}$ (hasta peso constante).

Se determinó la densidad aparente con un cilindro de $307,7 \mathrm{~cm}^{3}$ cada $10 \mathrm{~cm}$ de profundidad hasta los $30 \mathrm{~cm}$ de profundidad (Hillel, 1998). Se efectuaron 3 repeticiones por cada rango de profundidad y por cada tratamiento.

Se midió la infiltración del perfil mediante el método del anillo simple, con un infiltrómetro metálico de $21 \mathrm{~cm}$ de diámetro interno por $12 \mathrm{~cm}$ de alto y $3 \mathrm{~mm}$ de espesor. El anillo se clavó hasta una profundidad de $6 \mathrm{~cm}$ y se aplicó el agua con un frasco invertido (frasco de Mariotte) graduado en $\mathrm{mm}$ de lámina, dejando una carga hidráulica de $1,5 \mathrm{~cm}$ de altura. Se hicieron lecturas de entrada de agua cada 5 minutos hasta totalizar un período de una hora (tiempo en que la infiltración se estabilizó). Se registró la tasa de infiltración ( $\mathrm{mm} /$ hora) cada 30 minutos y la infiltración total (mm) (Elrick \& Reynolds, 1992). Se efectuaron 3 repeticiones por tratamiento.

Se determinó también la materia seca de la pastura de alfalfa con un anillo de $0,25 \mathrm{~m}^{2}$ de superficie. Se efectuaron 6 repeticiones por tratamiento. Una vez recogido el material, se llevó a estufa a $70^{\circ} \mathrm{C}$ hasta llegar a peso constante.

Para todas las variables evaluadas se realizó un análisis de la varianza y se efectuó la comparación de medias mediante el test de Tukey con un nivel de significancia del $5 \%$.

\section{RESULTADOS Y DISCUSIÓN}

En la Figura 1 se aprecian diferencias significativas en la resistencia a la penetración entre tratamientos hasta los $30 \mathrm{~cm}$. Estos resultados son coincidentes con los de Elisei et al. (2012); Álvarez et al. (2010), Cholaky et al. (2009); Colareda (2013) y Hilbert \& Pincu (2000) quienes concluyeron que los tratamientos con descompactación presentan menores valores de índice de cono que los testigos. Sólo en los dos primeros estratos se ha dado una mayor resistencia en el testigo lo cual podría ser atribuido al pisoteo animal que recibió el sustrato.

Se observa también que el aflojamiento producto del descompactado ha llegado hasta una profundidad superior $(30 \mathrm{~cm})$ que la de la labor $(25 \mathrm{~cm})$ en coincidencia con Colareda (2013) y Hilbert \& Pincu (2000). 


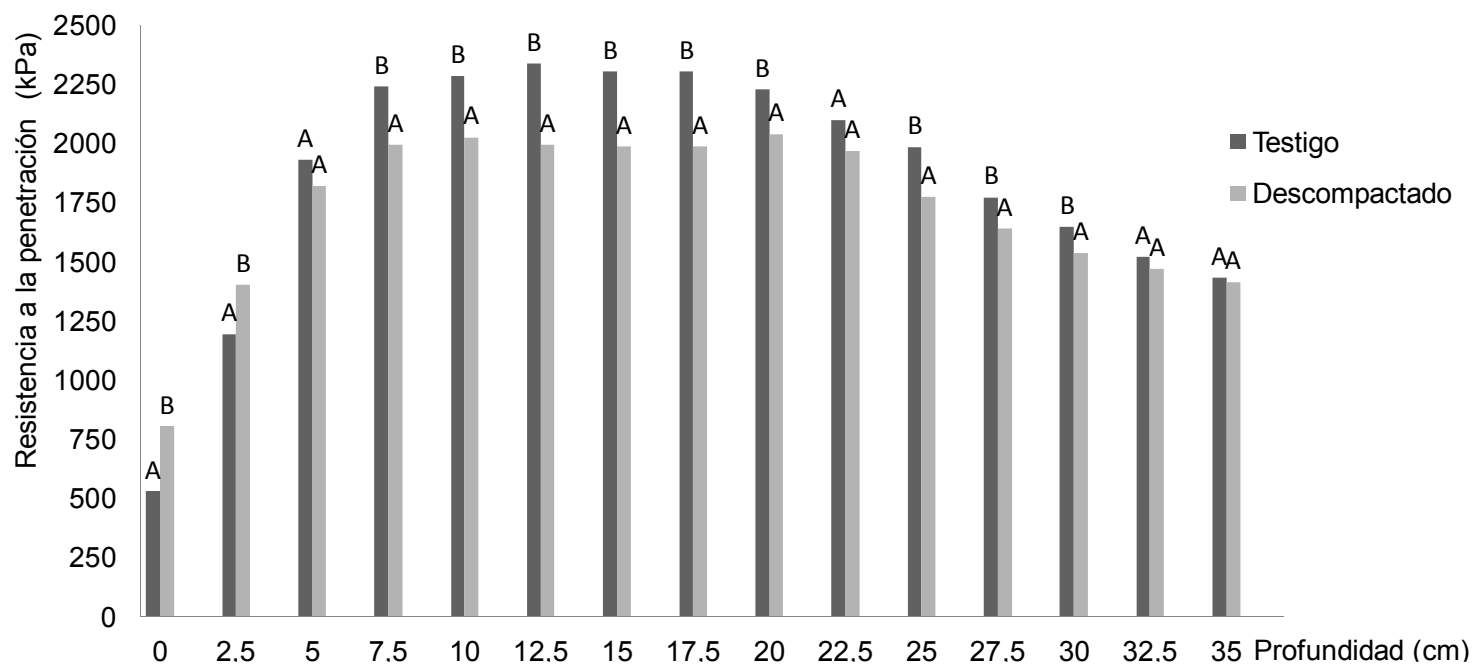

Figura 1. Resistencia a la penetración $(\mathrm{kPa})$ en intervalos de profundidad de $2,5 \mathrm{~cm}$ para ambos tratamientos. Letras iguales sobre las columnas denotan ausencia de diferencias mínimas significativas entre tratamientos según el test de Tukey $(p<0,05)$

El tratamiento testigo supera el valor de 2,0 MPa entre los 7,5 y $22,5 \mathrm{~cm}$ citado como límite a partir del cual las raíces de los cultivos dejan de crecer (Threadgill, 1982) mientras que se percibe en el descompactado un acercamiento a dicho valor en la misma zona como consecuencia de la reconsolidación en la profundidad trabajada.

Como puede observarse en la Tabla 1, las diferencias encontradas en la resistencia a la penetración no pueden ser explicadas por diferencias en la humedad gravimétrica relevada entre ellos.

En cuanto a la densidad aparente, no se encontraron diferencias significativas entre tratamientos en ninguno de los tres rangos de profundidad (Figura 2). Estos resultados no son coincidentes con lo encontrado por Soracco et al. (2005) quienes estudiaron sobre la misma serie de suelos, la incidencia de diferentes tratamientos mecánicos. La hipótesis explicativa estaría dada por la diferente humedad presente en ambas determinaciones, la cual pudo incidir en la adherencia entre suelo y cilindro muestreador, que termina modificando el volumen total de suelo que ingresa en cada probeta. Sin embargo, Alakukku (1996) y Botta et al., (2009b) concluyen que la densidad aparente es una variable menos sensible que la resistencia a la penetración a los efectos de cuantificar la compactación. Densidad Aparente (DA) y Resistencia a la Penetración (RP), son los dos parámetros más utilizados para relevar indirectamente el estado de compactación de un suelo (Jorajuría, 2005). Si bien el coeficiente de variabilidad de los datos de campo de la resistencia a la penetración suelen ser muy altos, pueden experimentalmente ser compensados por el gran número de repeticiones que pueden lograrse en la misma jornada sin que peligre perder la condición de humedad estable, necesaria para garantizar la biunivoquidad de los resultados. En cambio el relevamiento de la densidad aparente resulta ser mucho más lento y dificultoso, usándose modalmente tres repeticiones solamente para obtener un dato validable. No se han alcanzado los valores de densidad aparente limitantes de $1,65 \mathrm{Mg} / \mathrm{m}^{3}$ citados por Daddow \& Warrington (1983) Jorajuría (2005), Botta et al. (2010) ni los 1,46 a $1,63 \mathrm{Mg} / \mathrm{m}^{3}$ citados por Jones (1983), Tolon-Becerra et al. (2011) y Álvarez et al. (2010).

En cuanto al parámetro infiltración, en la Figura 3 se aprecia la homogeneidad observada entre ambos tratamientos. Estos resultados no son concordantes con lo citado por del Barrio (1984) quien encontró diferencias significativas en suelos con distintos manejos a lo largo de varios años. La pérdida del efecto de la descompactación con el tiempo percibida a través del parámetro infiltración sería coincidente con lo apreciado por Álvarez et al. (2006). Estos resultados también pueden corresponderse con lo cuantificado por Soracco et al. (2005) en cuanto a la relación entre los datos de densidad aparente e infiltración.

En la Figura 4 se aprecia la materia seca obtenida por hectárea en ambos tratamientos. El tratamiento descompactado duplica la masa del testigo, al igual que Tolon-Becerra et al., (2011); Marinello et al., (2017) y Jorajuría et al. (1997) quienes encontraron reducciones de rendimiento a causa de compactaciones inducidas. La persistencia del efecto de la descompactación sobre la variable rendimiento, coincide con Vepraskas et al. (1995) quienes manifiestan que los efectos del subsolado se mantienen dos años después de la labranza. Busscher et al. (1986) en cambio sostienen que el efecto se manifiesta sólo en las etapas tempranas del crecimiento de raíces, por la recompactación posterior que sufre el suelo. En el ensayo realizado, si bien el suelo descompactado adquiere nuevamente resistencia mecánica, se evidencia una diferencia de rendimiento de materia seca que justifica la labor aún dos años después de realizada, en coincidencia con lo manifestado por Barber (1994) quien determinó que si bien hay reconsolidación posterior a la labranza, la misma no afecta el crecimiento radicular tanto como el testigo sin laboreo. 
Revista de la Facultad de Agronomía, La Plata (2020) Vol 119 (1): 1-7

Tabla 1. Humedad gravimétrica (\%) en los distintos estratos evaluados.

\begin{tabular}{lccc}
\hline & $\mathbf{0 - 1 0} \mathbf{~ c m}$ & $\mathbf{1 0 - 2 0} \mathbf{~ c m}$ & $\mathbf{2 0 - 3 0} \mathbf{~ c m}$ \\
\hline Testigo & 24,07 a & $24,3 \mathrm{a}$ & $24,37 \mathrm{a}$ \\
Descompactado & $24,93 \mathrm{a}$ & $23,63 \mathrm{a}$ & $24,25 \mathrm{a}$ \\
\hline
\end{tabular}

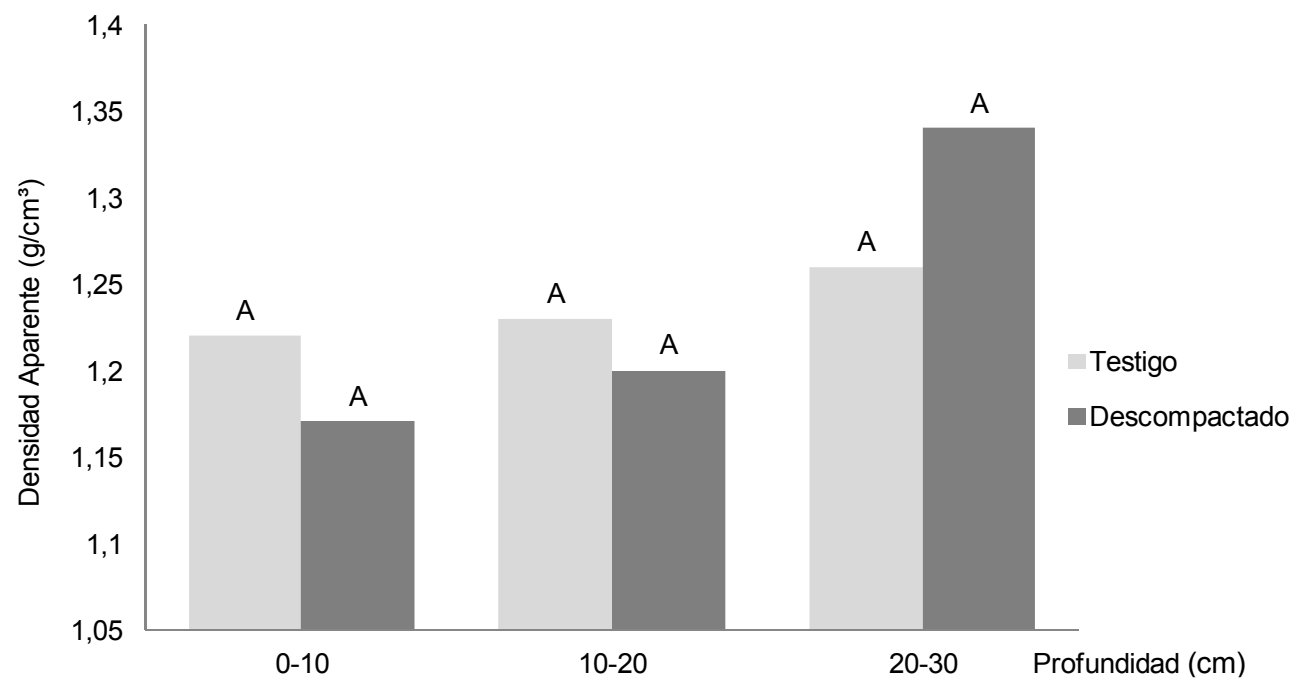

Figura 2. Densidad aparente $\left(\mathrm{g} \mathrm{cm}^{-3}\right)$ en los tres rangos de profundidades. Letras iguales sobre las columnas denotan ausencia de diferencias mínimas significativas entre tratamientos según el test de Tukey $(p<0,05)$.

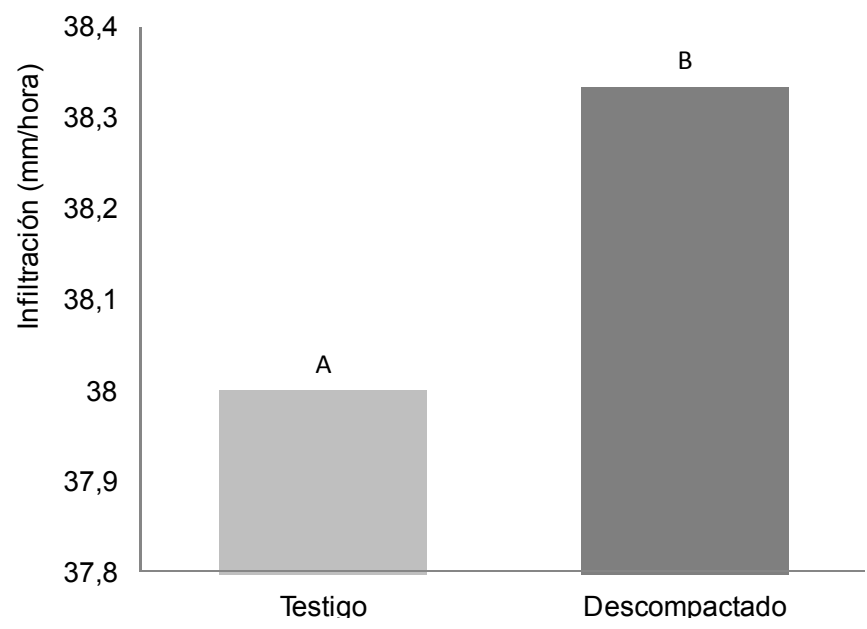

Figura 3. Infiltración de ambos tratamientos expresada en $\mathrm{mm} / \mathrm{h}$. Letras iguales sobre las columnas denotan ausencia de diferencias mínimas significativas entre tratamientos según el test de Tukey $(p<0,05)$.

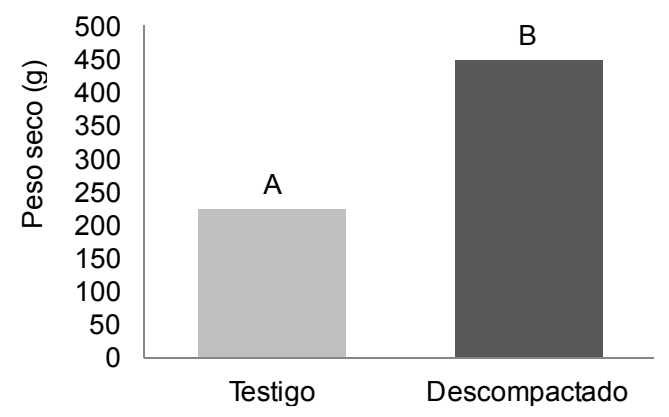

Figura 4. Materia seca de ambos tratamientos expresada en kilogramos/hectárea (kg/ha). Letras iguales sobre las columnas denotan ausencia de diferencias mínimas significativas entre tratamientos según el test de Tukey ( $p<0,05)$. 


\section{CONCLUSIONES}

La resistencia a la penetración evidencia los beneficios de la descompactación luego de dos años de realizada la labor.

Los efectos benéficos significativos de la descompactación sobre el rendimiento de materia seca son cuantificables aún dos años después de realizada la labor.

\section{BIBLIOGRAFÍA}

Alakukku, L. 1996. Persistence of soil compaction due to high axle load traffic. I Short- term effects on the properties of clay and organic soil. Soil and Tillage Research 37: 211-222.

Alakukku, L. 1997. Properties of fine-textured subsoils as affected by high axle load traffic. In: Academic Dissertation: Long term soil compaction due high axle load traffic.

Álvarez, C.R., M.A. Taboada, C. Bustingorri \& F.H. Gutiérrez Boem. 2006. Descompactación de suelos en siembra directa: efectos sobre las propiedades físicas y el cultivo de maíz. Ciencia del Suelo 24: 1-10.

Álvarez, C., C. Scianca, M. Barraco \& M. Díaz-Zorita. 2010. Contribución de los cereales invernales a la estructura del suelo en sistemas bajo siembra directa. XXII Congreso Argentino de la Ciencia del Suelo, Rosario. 10 pp.

Balbuena, H.R., A. Aragón, P. Mc Donagh, J. Claverie \& A. Terminiello. 1998. Effect of three different tillage systems on penetration resistance and bulk density. Proceedings of IV CADIR (Argentine Congress on Agricultural Engineering) 1: 197-202.

Barber, R.G. 1994. Persistence of loosened horizons and soybean yield increases in Bolivia. Soil Science Society of America Journal 58: 943-950.

Botta, G.F., A.T. Becerra \& F.B. Tourn. 2009a. Effect of the number of tractor passes on soil rut depth and compaction in two tillage regimes. Soil and Tillage Research 103(2): 381-386.

Botta, G.F., Tolon Becerra, A. \& Bellora Melcon, F. 2009b. Seedbed compaction produced by traffic on four tillage regimes in the rolling Pampas of Argentina. Soil \& Tillage Research 105 128-134

Botta, G.F., A. Tolon-Becerra, X. Lastra-Bravo \& M. Tourn. 2010. Tillage and traffic effects (planters and tractors) on soil compaction and soybean (Glycine max L.) yields in Argentinean pampas. Soil and Tillage Research 110: 167-174.

Botta, G.F., A. Tolón-Becerra, D. Rivero, D. Laureda, M. Ramírez-Roman, X. Lastra-Bravo, D. Agnes, I.M. Flores-Parra, F. Pelizzari \& V. Martirena. 2016. Compaction produced by combine harvest traffic: Effect on soil and soybean (Glycine max I.) yields under direct sowing in Argentinean Pampas. European Journal of Agronomy 74: 155-163.

Busscher, W.J., R.E. Sojka \& C.W. Doty. 1986. Residual effects of tillage on Coastal Plain soil strength. Soil Science 141: 144-148.

Cholaky, C., M. Caciavillani \& E. Bricchi. 2009. Persistencia del efecto de descompactadores en suelos con historia de siembra directa. Experiencia en Haplustoles/udoles del sur de Córdoba. Tercer Taller de
Física de Suelos, Universidad Nacional de Río Cuarto, Córdoba, Argentina. 8 pp.

Colareda, G.O. 2013. Persistencia de la descompactación en sistema de Siembra Directa. Tesis de grado. Facultad de Ciencias Agrarias y Forestales, UNLP, La Plata, Argentina. 42 pp.

Daddow, R.L. \& G.E. Warrington. 1983. Growthlimiting soil bulk densities as influenced by soil texture. USDA Forest Service, Fort Collins. pp.1-17.

Da Silveira Nicoloso, R, T.R. Carneiro Amado, S. Schneider, M.E. Lanzanova, V.C. Girardello \& J. Bragagnolo. 2008. Eficiência da escarificação mecânica e biológica na melhoria dos atributos físicos de um latossolo muito argiloso e no incremento do rendimento de soja. Revista Brasileira de Ciência do Solo 32(4).

del Barrio, R.A. 1984. Infiltración de agua en suelos de la Región Pampeana. Revista de la Facultad de Agronomía 5 (3): 183-191.

Elisei, J., B. Bonel, C. Irurtia, N. Gonzalez, C. Senigagliesi \& R. Mon. 2012. Efectos de diferentes escarificadores en propiedades físicas de suelo y enraizamiento del cultivo de maíz. Istro paper número 299. 19th Istro Conference. IV SUCS meeting. Striving for Sustainable High Productivity. Montevideo, Uruguay. $7 \mathrm{pp}$.

Elrick, D.E. \& W.D. Reynolds. (1992). Infiltration from constant-head well permeameters and infiltrometers. Advances in measurement of soil physical properties: Bringing theory into practice (advancesinmeasu). pp. 124.

Hamza, M.A. \& W.K. Anderson. 2005. Soil compaction in cropping systems. A review of the nature, causes and possible solutions. Soil and Tillage Research 82: 121145.

Hilbert, J.A. \& M. Pincu. 2000. Demanda energética de subsoladores Paratill. VI Congreso Argentino de Ingeniería Rural, II Congreso Americano de Educación en Ingeniería Agrícola. Departamento de Ingeniería Agrícola y Uso de la Tierra, Facultad de Ciencias Agrarias y Forestales, UNLP. La Plata, Argentina.

Hillel, D. (1998). Environmental soil physics: Fundamentals, applications, and environmental considerations. Elsevier.

INTA. 2010. Carta de Suelos de la República Argentina. Series de Suelo. Disponible en: http://anterior.inta.gov.ar/suelos/cartas/series/Los Nara njos.htm. Último acceso: marzo 2018.

Jones, C.A. 1983. Effect of soil texture on critical bulk density for root growth. Soil Science Society of America Journal 47:1208-1211.

Jorajuría, D., L. Draghi \& A. Aragón. 1997. The effect of vehicle weight on the distribution of compaction with depth and the yield of Lolium (Trifolium grassland). Soil and Tillage Research 41: 1-12.

Jorajuría, D. 2005. Compactación del suelo agrícola bajo tráfico. Una revisión. En: Reología del Suelo Agrícola Bajo Tráfico. Ed. EDULP, La Plata. pp. 39-55. Marinello, F., A. Pezzuolo, D. Cillis, A. Chiumenti \& L. Sartori. 2017. Traffic effects on soil compaction and sugar beet (Beta vulgaris L.) taproot quality parameters. Spanish Journal of Agricultural Research 15(1), 8 pp.

Nocelli, P.S. 2017. Estimación de superficie en siembra directa campaña 2016-2017. Asociación Argentina de Productores en Siembra Directa. Disponible en: 
http://www.aapresid.org.ar/superficie/. Último acceso: marzo 2018.

O'sullivan, M.F., E.A.G. Robertson \& J.K. Henshall. 1999. Shear effects on gas transport. Soil and Tillage Research 50: 73-83.

Soracco, G., T. Palancar \& S. Fajardo. 2005. Relación entre densidad aparente seca e infiltración del agua en el suelo. XIII jornadas de Jóvenes Investigadores de Universidades del Grupo Montevideo (AUGM). Universidad Nacional de Tucumán. pp. 162. Editado en CD.

Spoor, G., G.J. Tijink \& P. Weisskopf. 2003. Subsoil compaction: risk, avoidance, identification and alleviation. Soil and Tillage Research 73: 175-182.

Terminiello, A., J. Hilbert, J. Claviere, T. Palancar, L.
Draghi, R. Balbuena \& D. Jorajuria. 2007. Persistencia del efecto del escarificado en un suelo argiudol típico bajo siembra directa. CADIR, Córdoba, Argentina.

Threadgill, E.D. 1982. Residual tillage effects as determined by cone index. Transaction of the ASAE, St. Joseph. 25: 859-863.

Tolon-Becerra, A., X.B. Lastra-Bravo, G.F. Botta, M. Tourn, P. Linares, M. Ressia \& R. Balbuena. 2011. Traffic effect on soil compaction and yields of wheat in Spain. Spanish Journal of Agricultural Research 9 (2): 395-403. https://doi.org/10.5424/sjar/20110902235-10 Vepraskas, M.J., W.J. Busscher \& J.H. Edwards. 1995. Residual effects of deep tillage vs, no-tillage corn root growth and grain yield. Journal of Production Agriculture 8(3): 401-405. 